\title{
Las voces de los estudiantes y la educación histórica: oportunidades, tensiones y desafíos en la construcción de narrativas históricas con fragmentos autobiográficos
}

The voices of students and historical education: opportunities, tensions and challenges in the construction of historical narratives with autobiographical fragments

ANDRÉs FELIPE SOTO YONHSON ${ }^{\mathrm{a}}$

aUniversidad Alberto Hurtado, Santiago, Chile. 凶 andres.soto.y@gmail.com [orcid.org/0000-0001-5207-1423]

\section{RESUMEN}

Esta investigación didáctica analiza las voces presentes en los relatos elaborados por estudiantes sobre el antes, el durante y después de la dictadura cívico-militar, a medida que se implementó una secuencia didáctica que desarrollaba narrativas históricas con rasgos autobiográficos. La metodología de investigación es mixta con predominancia cualitativa. La muestra corresponde a trece narraciones elaboradas por un tercero medio de un establecimiento particular pagado ubicado en la comuna de Vitacura, en Santiago de Chile. Los resultados del análisis dan cuenta que las voces provenientes de las evidencias, del espacio familiar y de las y los estudiantes son diversas, presentan tensiones entre ellas y pueden ser modificadas por el material didáctico implementado. Situación que permite vincular el desarrollo de las habilidades del pensamiento histórico, el pasado reciente y los relatos autobiográficos de los y las estudiantes.

Palabra clave: Pensamiento histórico, cultura histórica, conciencia histórica, narrativas históricas, autobiografías.

\section{ABSTRACT}

This didactic research analyzes the voices present in the stories prepared by students about the before, during and after the civic-military dictatorship, as a didactic sequence that developed historical narratives with autobiographical features was implemented. The research methodology is mixed with a qualitative predominance. The sample corresponds to thirteen stories prepared in secondary education in a private school located in the municipality of Vitacura, in Santiago of Chile. The results of the analysis show that the voices coming from the evidence, the family 


\section{А. F. Sото}

space and the students are diverse, present tensions between them and can be modified by the teaching material implemented. Situation that allows to link the development of the skills of historical thinking, the recent past and the autobiographical accounts of the students.

Key words: Historical thinking, historical culture, historical consciousness, historical narratives, autobiographies.

\section{INTRODUCCIÓN}

La voz del estudiantado en las investigaciones desarrolladas desde la didáctica de la Historia y las Ciencias Sociales ha ocupado un rol secundario. En el mundo anglosajón y latinoamericano existe una importante tradición que ha investigado las dificultades que presentan los alumnos para desarrollar aprendizajes históricos y comprender el pasado, pero existen pocos trabajos que rescaten sus voces (Kitson, Steward \& Husbands, 2015; Plá \& Pagés, 2014). Un reflejo de ello, es el escaso conocimiento "sobre cómo niños y adolescentes captan el pasado en contextos menos formales" (Barton, 2010, p. 107), los cuales pueden determinar su comprensión sobre cómo funciona el mundo "y cómo es probable que se comporten las personas" (Kitson et al. 2015, p. 74).

Las investigaciones didácticas que han pretendido considerar las voces de los y las estudiantes han analizado sus narrativas, solo prestando atención al contenido histórico o a las habilidades históricas presentes en ellas (Barca, 2013; Cerri, De Oliveira \& Cuesta, 2014; Prego \& Xosé Armas, 2014). Según Plá y Pagés (2014), una investigación que evita la invisibilización del estudiante por el conocimiento científico es la desarrollada por Henríquez (2009), la cual demuestra que el aprendizaje "de la Historia no estaría determinado por el origen sociocultural de los alumnos sino por el desarrollo de las habilidades de pensamiento histórico que privilegien las formas que adquiere el discurso histórico" (Henríquez, 2009, p. 51). A pesar de ello, no se considera al alumno como joven, "como niño, como individuo cultural. En términos sociológicos, nos preocupamos de la socialización y no de la subjetivación” (Plá \& Pagés, 2014, p. 29)

Junto con lo anterior, existen investigaciones que buscan rescatar la voz de los y las estudiantes rastreando la conciencia histórica de los sujetos presente en sus narrativas. A partir de esto último, Schmidt (2016) concluye que los educandos consideran relevante la Historia, pero muy pocos logran usarla y relacionarla con sus actividades políticas fuera de la escuela. Por ello, plantea que hay una desarticulación entre el aprendizaje histórico escolar y la vida práctica de los alumnos. En esa misma línea, Plá y Pérez (2013) mencionan que "la educación informal parece ser más significativa que la educación formal en las formas de pensar y de recordar el pasado reciente" (p. 26).

La situación descrita permite evidenciar el escaso impacto que tiene el aprendizaje histórico en los educandos. Por lo general, solo citan algunas fechas o personajes sin demasiado sentido, por lo que son incapaces de comprender el significado o la trascendencia de un período histórico (Santiesteban \& Pagés, 2014), situación que supone "un obstáculo para utilizar el pasado como una especie de base a partir de la cual comentar el presente o el futuro" (Kitson et al. 2015, p. 164). Por ello, no es extraño que el pasado aprendido y 
las habilidades asociadas a este, no sean utilizadas por los jóvenes en su cotidianeidad. A partir de lo anterior, se desprende que existe una disociación entre la experiencia individual y social de los y las estudiantes con el proceso de enseñanza y aprendizaje de la Historia desarrollado en la escuela.

Considerando el problema didáctico mencionado, la pregunta de investigación que se pretendió responder fue: ¿cuáles son las transformaciones y/o continuidades de las voces presentes en las narrativas elaboradas por los y las estudiantes sobre el antes, el durante y el después de la dictadura cívico-militar en Chile, luego de ser expuestas a una unidad didáctica que pretende desarrollar narrativas históricas con fragmentos autobiográficos del estudiantado? Los objetivos que se desprenden de la interrogante anterior son:

- Caracterizar las voces presentes en las narrativas que los estudiantes elaboraron sobre el antes, durante y después de la dictadura civil militar chilena en la cultura histórica.

- Analizar las voces presentes en las narrativas históricas redactadas por las y los estudiantes durante la intervención didáctica.

- Analizar las voces presentes en las narrativas históricas con rasgos autobiográficos sobre el antes, durante y después de la dictadura cívico militar escritas al final de la intervención didáctica.

- Analizar las transformaciones y/o continuidades de las voces presentes en las narrativas elaboradas por los y las estudiante una vez finalizada la implementación didáctica.

\section{MARCO TEÓRICO}

El objetivo de investigación dialoga con cuatro conceptos. Estos son cultura histórica, conciencia histórica, narrativa histórica y narrativa autobiográfica.

El concepto de cultura histórica está relacionado con la práctica cotidiana de cada uno de los sujetos en un contexto histórico-social específico (Schmidt, 2014). Según Rusen (1992), este concepto circunscribe una serie de elementos, como son la creación artística, la lucha política por el poder, la educación escolar y extraescolar, el ocio y los procedimientos de la memoria pública, los cuales configuran el universo histórico-cultural de un lugar o espacio determinado en el que se desenvuelven las personas. En la cultura histórica siempre se encuentran en disputa múltiples narrativas y distintos enfoques, a través de los cuales se teje la conciencia histórica de los individuos y las identidades de los y las estudiantes (Sánchez, 2009; Cataño, 2011). Por ello, en los procesos de enseñanza y aprendizaje de la Historia desarrollados en el aula es necesario partir desde la cultura histórica en la que se desenvuelven cotidianamente los sujetos (Schmidt, 2014)

La conciencia histórica puede ser definida como "la suma de operaciones mentales con las cuales los hombres interpretan la existencia de evolución temporal de su mundo y de sí mismos, de modo que puedan orientar, intencionalmente, su vida práctica en el tiempo" (Plá \& Pagés, 2014, p. 23). En otras palabras, es la conciencia de la historicidad de cada uno 


\section{A. F. Sото}

de los sujetos con su entorno. Por ello, la "conciencia histórica se despliega entre lo que ha sucedido, lo que está sucediendo y lo que sucederá, lo cual hace referencia a cualquier aspecto de la vida de las personas" (Santisteban, 2010, p. 41).

Uno de los aspectos importantes de la conciencia histórica es la 'orientación temporal'. Esta puede desarrollarse en dos esferas de la vida. Una de ellas es la vida práctica, asociada con la temporalidad que circunscribe cada una de las acciones que los individuos desarrollan en el mundo social. La segunda se relaciona con la subjetividad interna, en la cual la temporalidad otorga una "autocomprensión y conocimiento de las características temporales dentro de las cuales aquéllos toman la forma de identidad histórica” (Rusen, 1992, p. 29). Precisamente, la identidad histórica "articula y entrecruza la dimensión temporal de un individuo, grupo, nación o comunidad, con la representación subjetiva de los sujetos y de sus cambios temporales en una dimensión personal ('yo'), y en una social ('nosotros')" (González \& Henríquez, 2003, p. 117).

La conciencia histórica-temporal "no se puede separar de la representación de la Historia" (Santisteban, 2010, p. 43). Esto quiere decir que las "operaciones por las cuales la mente humana realiza la síntesis histórica de las dimensiones del tiempo simultáneamente con las del valor y la experiencia, se encuentra en la narración: el relato de una historia" (Rusen, 1992, p. 29). Considerando estos elementos, la competencia narrativa puede definirse como "la habilidad de la conciencia humana para llevar a cabo procedimientos que dan sentido al pasado, haciendo efectiva una orientación temporal en la vida práctica presente por medio del recuerdo de la realidad pasada" (Rusen, 1992, p. 29).

La elaboración discursiva de una persona que ha desarrollado el pensamiento histórico debería expresarse "a través de la narración histórica y de la explicación causal e intencional" (Santisteban, 2010, p. 39). Las narraciones históricas deberían ser "una forma de explicación, por lo que la competencia narrativa significa construir explicaciones históricas, estructurales e intencionales, donde los personajes, los escenarios y los hechos históricos se sitúen en una trama coherente" (Plá \& Pérez, 2013, p. 16). A ellas se debería sumar la presencia de los elementos de propios de la Historia (Henríquez, 2013), ya que cualquier texto no puede denominarse como histórico, principalmente porque "la recreación de la época y el documento deben estar lo más apegados a la documentación que refleja la realidad que se presenta narrar" (Salazar, 2006, p.171).

El concepto que puede acercar las narrativas históricas construidas en la escuela con los relatos del estudiante es el de autobiografía. Para Delory-Momberger (2015) "la vida tiene lugar en el relato, y tiene lugar como historia" (p. 59). Por ello, los sujetos siempre realizan procesos de biografización, la cual es definida como "una dimensión del pensar y del proceder humano que, bajo la forma de una hermenéutica práctica, permite al individuo, en las condiciones de su inscripción socio-histórica, integrar, estructurar e interpretar las situaciones y los acontecimientos de su vivencia” (Delory-Momberger, 2015, p. 62). Esto es relevante considerarlo, ya que el mundo escolar ignora, descalifica u oculta una "gran parte de saberes experienciales de los que él mismo es el terreno, en provecho de los saberes formalizados a los que piensa como los únicos susceptibles de ser medidos, sancionados, validados" (Delory-Momberger, 2014, p. 707). 
Las investigaciones que se han centrado en las narrativas autobiográficas analizan cómo los sujetos "integran, estructuran, interpretan los espacios y las temporalidades de sus contextos histórico-culturales para examinar, por ese camino, el proceso de construcción del sujeto (o del grupo) en la interacción dialéctica entre el espacio social y el espacio personal" (Passegi, 2011, p. 29). Por ello, el trabajo con las autobiografías tiene un carácter formativo, ya que "la meta perseguida es contribuir a que los sujetos den sentido a su propia vida" (González, 2009, p. 210). A lo que se suma la posibilidad que tienen los individuos de reelaborar sus propios relatos, dándose cuenta que estos últimos no son una verdad inmutable, sino que dialogan constantemente con interpretaciones y cogniciones que potencian la reconstrucción de las narraciones autobiográficas y complejizan la autocomprensión de los sujetos. (Bolívar \& Domingo, 2006).

\section{METODOLOGÍA}

La intervención didáctica tuvo una duración de aproximadamente tres meses. Esta se realizó en un colegio particular de la comuna de Vitacura, en Santiago de Chile. Más específicamente, en las clases de Historia, Geografía y Ciencias Sociales que se desarrollaron en un tercero medio el año 2018. Fue seleccionado este nivel educativo porque el Programa de Estudio propuesto por el Ministerio de Educación (2015) se refiere temáticamente a un periodo todavía conflictivo y trascedente para la sociedad chilena, el cual se encontraba separado en dos unidades. La primera de ellas es el denominado periodo de transformaciones estructurales: Chile en las décadas de 1960 y 1970 y, la segunda, El quiebre de la democracia y la Dictadura Militar.

Si bien los participantes iniciales de la investigación fueron 32 estudiantes, solo se analizaron los trabajos que realizaron 13 de ellos, ya que desarrollaron todos los materiales didácticos propuestos y completaron las distintas etapas de la intervención en los tiempos asignados.

Las fases de la intervención didáctica que se considerarán para este artículo son tres. La primera de ellas, buscó caracterizar los contenidos y las estructuras de las narrativas que han elaborado los estudiantes del tercero medio en la cultura histórica. Para conseguir esto último, se les solicitó la confección de relatos, los cuales debían presentar un tema, una cronología o situación en el tiempo y un espacio delimitado. La actividad para recolectar estas narrativas se denominó ¿qué sé de Chile antes, durante y después del golpe de Estado de 1973? Junto con ello, debían solicitar a dos personas significativas para ellos que realizaran el mismo ejercicio. La segunda fase corresponde a la intervención didáctica, la cual tuvo como objetivo desarrollar las habilidades del pensamiento histórico presentes en las narrativas históricas, destacando la continuidad y cambio desde una perspectiva social e individual (autobiográfica), las cuales serían progresivamente aplicadas en los relatos construidos inicialmente. En la última fase los estudiantes debieron evaluar y reelaborar las narraciones realizadas al inicio de la intervención didáctica, a partir de las habilidades históricas enseñadas, considerando las redacciones de sus personas significativas y los fragmentos autobiográficos trabajados con anterioridad. 


\section{A. F. Sото}

Esta investigación procesual es de carácter mixto pero con peso en lo cualitativo. Por ello, este proceso investigativo no pretendió probar hipótesis construidas de antemano, sino que estas se fueron generando "durante el proceso y van refinándose conforme se recaban más datos” (Hernández, Fernández \& Baptista, 2010, p. 9). A partir de esta premisa, se busca resaltar que esta intervención didáctica se aplicó "sobre una cultura existente, sobre una lógica muy potente, que las interpreta, las rechaza o, a veces las asimila” (Merchán, 2010, p. 106). De ahí que la "realidad se define a través de las interpretaciones de los participantes en la investigación respecto de sus propias realidades" (Hernández et al. 2010, p. 9), las que "van modificándose conforme transcurre el estudio y son las fuentes de datos" (Hernández et al. 2010, p. 9).

La información recolectada de las narraciones confeccionadas por los estudiantes antes, durante y después de la intervención didáctica fue analizada teniendo como referencia el análisis de contenido, el cual puede ser definido como una técnica de investigación que busca formular, a partir de ciertos datos, inferencias reproducibles y válidas que puedan aplicarse a su contexto (Krippendorff, 1990). Considerando esto último, en cada una de las tres fases de la intervención fueron emergiendo distintos códigos y se construyeron categorías utilizando el programa Atlas-Ti, los cuales, en una última instancia, fueron comparados para identificar las categorías que se transformaron y/o continuaron en el final de la implementación didáctica. Para enriquecer el análisis se consideraron aspectos cuantitativos, los cuales están relacionados con la obtención de frecuencias y magnitudes de las categorías que fueron emergieron en el análisis, aportando un "mayor entendimiento, ilustración o clarificación de los resultados" (Hernández et al. 2010, p. 551).

\section{RESULTADOS}

\section{Antes de la intervención didáctica}

En esta sección se caracterizan las voces presentes en las trece narrativas que las y los estudiantes elaboraron sobre el antes, durante y después de la dictadura civil militar en Chile considerando solo su cultura histórica. En la totalidad de relatos analizados se encontraron fragmentos donde el narrador es omnisciente, situación que provoca que la voz del estudiante se difumine en un supuesto manto de objetividad histórica. A pesar que el grupo estudiantil utiliza mayoritariamente esta forma de relatar, su voz se encuentra explícita en algunos fragmentos de las narraciones (ver Tabla 1).

La categoría con menor cantidad de fragmentos es la denominada 'yo asociado con el conocimiento histórico'. En ella, las y los estudiantes se distancian del saber histórico porque "lo único que sé acerca de la Dictadura lo aprendí en clases de Historia y, por historias que escucho de mis amigos y familiares" (Estudiante 2). Junto con lo anterior, señalan al inicio de su relato que "en las próximas páginas se verá un punto de vista más subjetivo de parte del narrador" (Estudiante 10). En estos tres extractos se relaciona el conocimiento histórico con la objetividad, por ello la voz del estudiante emerge en el relato para señalar su escaso manejo sobre este proceso histórico. Esto demuestra que los estudiantes esperan que las clases de Historia cuenten la verdad sobre lo que ocurrió en el pasado. 
LAS VOCES DE LOS ESTUDIANTES Y LA EDUCACIÓN HISTÓRICA

Tabla 1. Categorías que presentan la voz de los y las estudiantes.

\begin{tabular}{cccc}
\hline Categorías & $\begin{array}{c}\text { Yo asociado con el } \\
\text { conocimiento histórico }\end{array}$ & $\begin{array}{c}\text { Yo asociado con el conocimiento social } \\
\text { (conexiones pasado-presente) }\end{array}$ & $\begin{array}{c}\text { Yo como evaluador } \\
\text { del pasado }\end{array}$ \\
\hline $\begin{array}{l}\text { Cantidad de } \\
\text { fragmentos }\end{array}$ & 3 & 8 & 26 \\
\hline
\end{tabular}

Fuente: Elaboración propia.

La segunda categoría con mayor cantidad de fragmentos es el 'yo asociado con su presente social (conexión pasado-presente)'. En esta se realizan distintas reflexiones sobre la importancia y/o las consecuencias del proceso histórico estudiado, las cuales interrumpen al narrador omnisciente y los educandos se incorporan como una voz distinta en el relato. Por ejemplo, mencionan que "lo único relevante de pasar es que no hay que olvidar lo sucedido ni lo que está sucediendo en el presente" (Estudiante 10) porque "lo que si hay que tener en claro es que NADA justifica las torturas que se efectuaron durante la dictadura, sea cual sea el propósito" (Estudiante 20). Junto con lo anterior, dos fragmentos se refieren al rencor que la sociedad heredó de este periodo histórico, mencionando que "era algo que tenía que pasar y ya pasó bastante tiempo para que gente socialista o comunista siga marcando o recordando esta fecha y queriendo arrestar a militares que la mayoría ya están muertos" (Estudiante 22).

La última categoría identificada en las narraciones iniciales de los estudiantes es la que mayor frecuencia presenta, específicamente 26 fragmentos, y fue denominada 'yo como evaluador del pasado', la cual se asocia con los códigos presentes en la Tabla 2.

Tabla 2. Evaluación de temáticas históricas con la voz de los y las estudiantes.

\begin{tabular}{lcccc}
\hline Códigos & $\begin{array}{c}\text { Contexto histórico } \\
\text { del gobierno de } \\
\text { Salvador Allende }\end{array}$ & $\begin{array}{c}\text { Reformas de Salvador } \\
\text { Allende y violaciones a } \\
\text { los Derechos Humanos }\end{array}$ & $\begin{array}{c}\text { Dictadura } \\
\text { cívico-militar de } \\
\text { Augusto Pinochet }\end{array}$ & $\begin{array}{c}\text { Vuelta a la democracia } \\
\text { y gobiernos que } \\
\text { antecedieron a la } \\
\text { Unidad Popular }\end{array}$ \\
\hline $\begin{array}{l}\text { Cantidad de } \\
\text { fragmentos }\end{array}$ & 7 evaluaciones & 6 evaluaciones $(\mathrm{c} / \mathrm{u})$ & 5 evaluaciones & 1 evaluación (c/u)
\end{tabular}

Fuente: Elaboración propia.

El código denominado 'contexto histórico del gobierno de Salvador Allende' es donde más evaluaciones se evidencian y las palabras que visibilizan la posición de los estudiantes son contexto complicado, problemas, crisis, caos y desastre. Por ejemplo, mencionan que "presidente no la tuvo fácil, ya que tuvo que enfrentar problemas como la huelga de los camioneros, lo que ocasionó un gran problema a la economía del país" (Estudiante 9).

En un segundo lugar, las voces de los y las estudiantes se encuentran presentes cuando evalúan las reformas del gobierno de Salvador Allende. Estas son catalogadas como cambios drásticos, inicio de los problemas, producen polarización política o que no mejoran la economía. Situación que se expresa cuando señalan que "él [Salvador Allende] fue alguien con ideales socialistas y trató de implementar cambios muy drásticos en el país, lo cual causó que la gente se dividiera entre partidarios y opositores" (Estudiante 2). 


\section{А. F. Sото}

La tercera temática en donde la voz del alumno emerge y evalúa el pasado son las violaciones a los derechos humanos llevadas a cabo durante la dictadura cívico-militar. Los fragmentos se relacionan directamente con Augusto Pinochet, ya que es sindicado como el que realizó las violaciones. Esto se evidencia cuando mencionan que "En todos estos años Pinochet exilió, asesinó y torturó a muchísima gente por el simple hecho de pensar diferente, entonces, aunque haya activado la economía, un porcentaje de chilenos no estaban a favor de sus medidas para gobernar Chile, por eso mismo se conoce este gobierno como Dictadura Militar" (Estudiante 15).

\section{Durante la intervención didáctica}

Si bien la intervención didáctica tuvo como objetivo desarrollar de forma progresiva una serie de habilidades del pensamiento histórico, en esta oportunidad solo fueron analizadas las voces que se encuentran presentes en las explicaciones históricas que elaboraron los estudiantes. El material didáctico que pretendió desarrollar la habilidad antes mencionada, incorporó cuatro fuentes secundarias relacionadas con la Constitución de 1980, las cuales tenían que ser utilizadas obligatoriamente y de forme explícita por los y las estudiantes para elaborar sus respuestas. Del análisis de las explicaciones históricas elaboradas emergieron cuatro categorías principales (ver Tabla 3).

Tabla 3. Voces presentes en las explicaciones de los y las estudiantes.

\begin{tabular}{lcccc}
\hline \multicolumn{1}{c}{ Categorías } & Sin agentividad & $\begin{array}{c}\text { Citas textuales o } \\
\text { parafraseo con } \\
\text { referencias }\end{array}$ & $\begin{array}{c}\text { Interpretación de } \\
\text { las fuentes }\end{array}$ & $\begin{array}{c}\text { Reflexión } \\
\text { de los y las } \\
\text { estudiantes }\end{array}$ \\
\hline $\begin{array}{l}\text { Número de } \\
\text { explicaciones }\end{array}$ & 4 & 9 & 11 & 16 \\
\hline
\end{tabular}

Fuente: Elaboración propia.

Las explicaciones que corresponden a la categoría denominada sin agentividad son cuatro y corresponden al 10\% del total. En ellas, el narrador es omnisciente, lo cual se relaciona con las dificultades presentadas por los estudiantes para reconocer los personajes y/o cuasipersonajes históricos que producen una acción. De ahí que estas explicaciones pretendan ser escritas desde la objetividad. Esto se encuentra expresado cuando se menciona que con la Constitución de1980 "se hace público el proyecto de instaurar una democracia protegida con la idea de que el régimen de Pinochet se extendiera dejando una obra social, económica e institucional para Chile" (Estudiante 21).

Las tres categorías restantes donde emergen voces distintas, sin la presencia de un narrador omnisciente, corresponden al 90\% de las explicaciones. Todas ellas tienen una característica en común, los estudiantes identifican claramente, como mínimo, un personaje o cuasipersonaje que realiza una o más acciones en un contexto determinado y con finalidades específicas.

Las explicaciones categorizadas como citas o parafraseo con referencias corresponden al 23\% del total. En ellas, los estudiantes incorporan la voz de las fuentes secundarias, ya 
que citan la información textualmente o bien mencionan de qué fuente secundaria fueron extraídas las ideas que utilizan. Esto se evidencia cuando un alumno señala que:

Siendo Pinochet el agente de acción histórico 4, tuvo como intención "proyectar la idea de que el régimen iba a extenderse más allá de su persona y del gobierno militar", según la cita se puede entender que Pinochet crea esta constitución para dejar algo del régimen para cuando este terminara y que este involucraba a todos, no solo a él y su gobierno. (Estudiante 15).

En la categoría denominada interpretación de las fuentes, las cuales corresponden al 28\% del total de explicaciones, los estudiantes elaboran una mixtura entre su voz y la presente en las fuentes secundarias sin incorporar alguna referencia. Por ejemplo, esto ocurre cuando señalan que Pinochet pensaba que la "única manera de tener una democracia era manteniendo a raya al comunismo. Por lo cual la democracia protegida se ocupa para reprimir a los opositores del régimen, ya que estos son una amenaza contra el sistema que se quiere implementar" (Estudiante 2). En este fragmento se aprecia la interpretación de una parte de una de las fuentes secundarias propuestas en el material. El problema de esto último, son las simplificaciones. Esto ocurre con el concepto de democracia protegida, ya que no se consideran las ideas relevantes como es el enclaustramiento de la ciudadanía en el ámbito doméstico apartándola de la esfera pública. Solo se relaciona el concepto de democracia protegida con Pinochet y es entendida como una forma de controlar y eliminar a los opositores.

La última categoría que emergió en las explicaciones de los estudiantes fue la denominada reflexiones personales, las que corresponden al $40 \%$ del total. A pesar que el material didáctico explícitamente solicitaba el uso de las fuentes secundarias para sustentar sus explicaciones, en ninguno de estos relatos se encuentra esto último. Sin duda, se evidencian ciertas resistencias a la incorporación de referencias, aunque no de su uso para la construcción de reflexiones personales. A pesar de esto, las explicaciones que corresponden a esta categoría identifican correctamente al personaje o cuasipersonaje que realiza la acción y la finalidad de estas. Lo nuevo, en comparación con las categorías anteriores, es la incorporación de reflexiones de los estudiantes, como, por ejemplo, se muestra a continuación:

Cuando Pinochet instaura el término democracia protegida lo que hace es intentar hacer sentir al pueblo que se encuentran en un país en el cual sus opiniones son 100\% válidas para el gobierno, y donde lo que se hace en Chile es en función del pueblo, pero lo que realmente ocurre es que esta "Democracia", es la manera que Pinochet utiliza para comenzar a dejar todo a su favor, como la constitución, senadores vitalicios, etc. (Estudiante 20)

\section{Al final de la intervención didáctica}

En los trece relatos finales de los estudiantes que fueron analizados en esta investigación se identificaron distintas voces, ya que en todas las narraciones se presenta un narrador omnisciente y diferentes voces alternativas (ver Tabla 4). 


\section{А. F. Sото}

En los trece relatos finales se evidencia, al menos, una sección con un narrador omnisciente, lo cual no se concentra en una temática específica, sino que se expresa en las transformaciones socialistas del gobierno de la Unidad Popular, las violaciones a los derechos humanos o la denominada vuelta a la democracia. Este tipo de voz presente en las narraciones se encuentra reflejada en la siguiente cita sobre el gobierno de Salvador Allende, el cual "logró llevar a cabo varios cambios que se plantearon durante la época de propuestas en las elecciones, como lo es: La reforma agraria, la revolución por la vía diplomática y la equidad en algún sentido de la palabra”. (Estudiante 10).

Tal como se aprecia en el fragmento anterior, se narran distintos hechos del pasado a partir de una voz, la cual relata desde una perspectiva objetiva el proceso histórico estudiado. Esta forma de narrar no predomina, sino que dialoga constantemente con distintas voces, como son las provenientes de las fuentes históricas, las personas significativas de los y las estudiantes o del mismo alumno.

Tabla 4. Voces presentes en las narraciones finales de los y las estudiantes.

\begin{tabular}{|c|c|c|c|c|}
\hline $\begin{array}{l}\text { Tipo de voces } \\
\text { presentes en los } \\
\text { relatos }\end{array}$ & $\begin{array}{l}\text { Relatos con al menos } \\
\text { una sección con } \\
\text { narrador omnisciente }\end{array}$ & $\begin{array}{l}\text { Relatos con secciones } \\
\text { que incorporan las } \\
\text { voces presentes de } \\
\text { fuentes primarias o } \\
\text { secundarias }\end{array}$ & $\begin{array}{c}\text { Relatos con secciones } \\
\text { que incorporan la } \\
\text { voz de sus personas } \\
\text { significativas }\end{array}$ & $\begin{array}{c}\text { Relatos con secciones } \\
\text { que incorporan la } \\
\text { voz del estudiante }\end{array}$ \\
\hline $\begin{array}{l}\text { Cantidad de } \\
\text { narraciones }\end{array}$ & 13 & 2 & 12 & 13 \\
\hline
\end{tabular}

Fuente: Elaboración propia.

Del total de relatos finales solo en dos de ellos los educandos incorporan una voz proveniente de las fuentes primarias y/o secundarias. Situación que es una constante en las distintas etapas de la intervención didáctica, lo cual nos entrega indicios de las resistencias de los estudiantes a la hora de incorporar fuentes históricas en la elaboración de sus narraciones sobre este pasado conflictivo. En esta ocasión, solo utilizaron un extracto de la Constitución de 1980 trabajado en las clases anteriores e incorporaron la definición de dictadura utilizando la Real Academia Española (RAE).

En comparación con la categoría anterior, doce de los trece relatos finales utilizaron extractos de las narraciones realizadas por personas significativas para los estudiantes sobre el periodo estudiado. Estas se concentran en dos momentos históricos trabajados durante la intervención. El primero son los problemas económicos que tuvo el gobierno de la Unidad Popular, más específicamente, los relacionados con la inflación y las filas para conseguir alimento que fueron consecuencia del desabastecimiento del periodo. La segunda temática histórica donde incorporan las experiencias de personas significativas para ellos, es el golpe de Estado del 11 de septiembre y las distintas consecuencias que tuvo para la población. De este tipo fueron encontrados cinco extractos, evidenciados con el siguiente fragmento:

Como por ejemplo muchas personas de Temuco como me contó mi abuela salieron a celebrar y a darle las gracias a los militares por intervenir el gobierno socialista de 
Allende, mientras que mi abuela que vivía en Santiago y trabajaba en el paseo Bulnes tuvo que correr para poder resguardar su vida y así empezó la dictadura. (Estudiante 1).

A estos dos temas anteriores, los cuales fueron los más recurrentes, los siguen fragmentos asociados a la economía durante la dictadura de Augusto Pinochet, los aspectos positivos del gobierno de la Unidad Popular y, finalmente, las reacciones a las votaciones del Sí y del No de 1988.

Además de lo anterior, en la totalidad de los relatos finales se identificó la voz del estudiante, el cual se incorpora como una segunda voz, quebrando la objetividad presente en los relatos con un narrador omnisciente. Estas se expresan en cinco momentos diferentes, los cuales se pueden apreciar en la Tabla 5.

Tabla 5. Categorías que presentan la voz del estudiante.

\begin{tabular}{lcccc}
\hline Categoría & $\begin{array}{c}\text { Evaluación del } \\
\text { pasado }\end{array}$ & $\begin{array}{c}\text { Presente } \\
\text { individual de } \\
\text { estudiante }\end{array}$ & $\begin{array}{c}\text { Futuro (colectivo } \\
\text { e individual) }\end{array}$ & $\begin{array}{c}\text { Presente social del } \\
\text { estudiante }\end{array}$ \\
\hline $\begin{array}{l}\text { Cantidad de } \\
\text { fragmentos }\end{array}$ & 6 & 10 & 14 & 20 \\
\hline
\end{tabular}

Fuente: Elaboración propia.

Del total de narraciones elaboradas, se identificaron seis fragmentos, los cuales corresponden al 12\% del total de esta sección, en donde las y los alumnos se incorporaron como otra voz, la cual no es objetiva ni omnisciente, evaluando las violaciones a los derechos humanos que se realizaron durante la Dictadura Militar en Chile. Esto último se evidencia cuando mencionan que "el inicio de la dictadura fue el más brutal creo yo, ya que se implementaron diversos métodos de control y 'extirpación del comunismo', eliminando poblaciones comunistas y manteniendo un control rígido sobre todo el pueblo chileno" (Estudiante 2).

Otra categoría donde emerge parcialmente la voz de los educandos es la denominada 'presente social del estudiante'. Ella se presenta en veinte fragmentos de las narraciones finales, correspondiendo al 40\% de esta sección, los cuales presentan aspectos autobiográficos, pero no de forma explícita, ya que no aparece el sujeto en primera persona, sino que relata y se mimetiza en la pluralidad. En ellos mezclan su experiencia personal con las formas de actuar y de pensar de toda la sociedad. De ahí fueron identificados algunos fragmentos relevantes, entre los que destaca, por ejemplo, cuando mencionan que una de las ideas que viene de la Dictadura Militar son los "pensamientos estereotipos para algunas clases sociales y personas. O bien, el pensar que todo lo que intervenga el Estado es algo de izquierda, no necesariamente que el Estado intervenga en algunas cosas del país significa que esas cosas sean tiradas para el lado de la izquierda" (Estudiante 10).

Además de lo anterior, un 20\% de fragmentos que presentan voces de los estudiantes de forma explícita, en las cuales no se sumergen o mimetizan con los aspectos sociales del presente y se aprecian aspectos autobiográficos. Entre ellos resaltan, por ejemplo, cuando mencionan que provocó "en mi una indiferencia con el tema de la política, ya que mi familia 


\section{A. F. Sото}

aprendió a vivir con ello y a respetar las reglas” (Estudiante 17). Quizás el fragmento que más representa a esta sección es el siguiente:

Durante la dictadura se implantaron muchas formas de pensar y actuar en los chilenos, formas de pensar que hasta yo tengo y nunca me había dado cuenta, estas son, por ejemplo, aquella idea de la sociedad no debía ser política o que todo aquello que viene del Estado es malo, estas ideas no solo se encuentran en mi cabeza sino en las de muchos chilenos como mis familiares o muchas personas con las que me relaciono día a día. Estas ideas continúan en la cotidianidad de muchos chilenos por ejemplo cuando la juventud no va a votar solo por el hecho de que no les interesa la política, o cuando creemos que un colegio, liceo, es malo solo por ser estatal, también la idea de que la sociedad es individualista como cuando los sindicatos pierden credibilidad, o solo se piensa en el bien individual. Todas estas ideas, y más, se implantaron durante el golpe militar. (Estudiante 3).

Las últimas dos secciones, las cuales corresponden al 28\% de fragmentos, donde emerge la figura del sujeto que narra están relacionados con el futuro de nuestro país, como comunidad, y con las acciones que realizaría el estudiante para construir el futuro.

En esta ocasión la voz del estudiante emerge cuando se relaciona con el futuro colectivo de Chile. De ahí que mencionen que este país "necesita dejar atrás el miedo y el resentimiento que la tiene de la década de los sesenta, setenta y ochenta”, lo cual se lograría a partir de la frase “Aceptar es madurar", la cual exige "No tanto división de derecha o izquierda, sino más unidad para alcanzar una meta juntos" (Estudiante 10).

El último aspecto que permite el surgimiento del sujeto se relaciona con las acciones que los estudiantes podrían hacer para construir el futuro que pensaron para Chile. Para esto último mencionan que "lo que yo planteo es que la gente compartiera sus relatos de lo que sucedió en la Dictadura para que de esta forma no se vuelva un tema olvidado" (Estudiante 1). O bien, otros señalan que lo que realizarían sería "relacionarme con gente de todos los estratos sociales para así hacerles dar cuenta de cuáles son las realidades que viven en Chile, de esa forma ellos se darán cuenta de la sociedad que existe”. A lo que agrega que participará "en movimientos y marchas para generar cambios reales” (Estudiante 18).

\section{ANÁLISIS DE LOS RESULTADOS}

Al analizar los relatos de los y las estudiantes en las tres etapas consideradas en esta investigación, se pudo identificar que un aspecto que persiste, es que todos ellos utilizan un narrador omnisciente. Es decir, la voz del estudiante no se encuentra explícitamente presente, ya que este último pretende contar objetivamente el pasado. Esta forma de narración "es común en los manuales de Historia, así como en los libros de texto de Historia y Ciencias Sociales del sistema escolar" (Henríquez et al. 2018, p. 30). Por ello, se construyen relatos que "no tienen o no reconocen voces o posiciones alternativas" (Henríquez, Carmona, Quinteros \& Garrido, 2018, p. 29). Esta situación demuestra la existencia de una percepción 
estudiantil particular sobre la disciplina histórica, en donde la objetividad sería su principal característica.

Las narraciones que presentan un sentido histórico más complejo incorporan distintas voces en un modo ondulante en el mismo texto (Henríquez et al. 2018). Justamente, la incorporación de voces alternativas, las cuales tensionan o complementan lo mencionado por el narrador omnisciente, como pueden ser el parafraseo, las citas textuales o la aparición de la voz de los y las estudiantes que narran, serán analizadas con detalle a continuación.

En primer lugar, un aspecto que se modifica es la aparición de la voz del estudiante en las narraciones evidenciando la desigualdad entre sus conocimientos y los contenidos históricos relacionados con el gobierno de la Unidad Popular y la dictadura cívico militar. En la etapa inicial de la intervención tres alumnos señalan que no tienen los conocimientos necesarios para realizar la narración, por ello, lo contarán desde una perspectiva subjetiva. Lo que demuestra esta pequeña muestra es que no consideran válidos para el aula los conocimientos que podrían haber adquirido en otras instancias de aprendizaje, a lo que se suma la escasa conciencia de los distintos espacios educativos informales que influyen en su desarrollo. Esto último fue trabajado durante la intervención didáctica, lo cual tuvo como consecuencia que en la fase final no declararan la asimetría entre lo que deberían saber del contexto histórico estudiado y sus conocimientos. El problema es que vuelven a emerger cuando se refieren a los años noventa en Chile, señalando, por ejemplo, que "después del golpe militar, me repito al igual que en mi anterior relato, no sé mucho, solo que fue Aylwin quien asumió el poder luego de que el NO ganara en el plebiscito". (Estudiante 3).

En segundo lugar, un aspecto recurrente durante toda la intervención didáctica es la resistencia del grupo estudiantil a incorporar fuentes históricas, tanto primarias como secundarias, en las narraciones que confeccionan. En los relatos iniciales no se identificó ningún fragmento que las utilizara como una voz alternativa o complementaria al narrador omnisciente de los textos. Si bien esta situación cambia sutilmente en la etapa de aplicación, en las narraciones finales solo dos de los trece estudiantes incorporaron respaldos en sus relatos.

A raíz de lo anterior, es interesante considerar el concepto de evidencialidad planteado por Plá (2005), el cual está asociado con la verificalidad propia del oficio de los historiadores, a través del uso de fuentes históricas que le otorgan autoridad al escrito. Las escasas evidencias incorporadas por el grupo estudiantil, si bien presentan una segunda voz, restringen la interpretación y la apropiación de estas últimas por los educandos. La consecuencia de esto último es que el alumno no tiene la posibilidad de desarrollar el pensamiento crítico, ya que "al sopesar sus propios argumentos se niega a sí mismo como posible autor de la interpretación histórica" (p. 149). Por ello, "la verdad histórica no está, por tanto, en su interpretación [la del estudiante], sino que en el principio de autoridad del historiador" (p. 149). En esta misma línea, lo didácticamente problemático es que las explicaciones históricas que incorporaban reflexiones de los estudiantes, sin considerar evidencias, estaban más cercanas a la habilidad de explicar históricamente. El problema de estas últimas era su nula verificabilidad, ya que eran desarrolladas sin considerar las evidencias y, quizás lo más relevante, lo que escribían los y las estudiantes no rivalizaba con el principio de autoridad presente en las fuentes históricas, ya que no eran explícitamente consideradas. 


\section{A. F. Sото}

En tercer lugar, un aspecto nuevo es que las voces que más incorporan los estudiantes en sus relatos finales son las provenientes de fragmentos realizados por sus personas significativas sobre el antes, el durante y después de la dictadura cívico-militar. De hecho, en doce de las trece narraciones finales realizan esto último. Estas se concentran en dos temáticas conflictivas del proceso histórico. La primera son los problemas económicos que tuvo el gobierno de la Unidad Popular, precisamente los asociados con la inflación y las llamadas 'colas' para adquirir alimentos. La segunda, fueron las consecuencias del golpe de Estado para las personas que vivieron en ese contexto. Esta situación tensiona el principio de autoridad de las fuentes históricas porque estas últimas, para este grupo de estudiantes, son menos relevantes que las narraciones de sus personas significativas sobre el pasado conflictivo y reciente de Chile. De hecho, los fragmentos elaborados por sus personas importantes se insertan en los relatos como respaldo o complemento a lo mencionado por el narrador omnisciente. Por lo tanto, no se posicionan como una voz alternativa a lo relatado. Esto último refleja la tensión que se puede encontrar presente en las aulas a la hora de trabajar didácticamente este pasado, ya que las evidencias entregadas por los docentes podrían chocar o contradecir las narraciones provenientes del ámbito familiar u otros espacios significativos para los y las estudiantes.

En cuarto lugar, las voces de los y las estudiantes sufren transformaciones a la hora de incorporarse en sus narraciones durante la intervención didáctica. Esto ocurre con las evaluaciones a partir de temáticas conflictivas y en las relaciones pasado, presente y futuro, desde una perspectiva individual y social.

Las evaluaciones presentes en las narraciones, en las cuales emerge la voz de la y el estudiante, se circunscriben a temáticas conflictivas relacionadas con la idea de cambio, la cual es percibida por los educandos como la causante de inestabilidades sociales, económicas o políticas. En los relatos iniciales fueron identificados 26 fragmentos que presentaban las características anteriores, los que se concentraron en las reformas económicas y sociales de carácter socialista llevadas a cabo por el gobierno de Salvador Allende y en las violaciones a los derechos humanos que se produjeron durante la dictadura cívico-militar. En las narraciones finales disminuyen a seis la cantidad de evaluaciones y, por lo tanto, la presencia de la voz del estudiante con características evaluativas se fue difuminando, lo cual podría deberse a su incorporación en el presente y el futuro, desde una perspectiva individual y social, dejando el pasado en manos del narrador omnisciente y los fragmentos de sus personas significativas.

Además de lo anterior, el análisis de las narraciones demuestra un aumento significativo en las vinculaciones entre el pasado y el presente social de los y las estudiantes, más específicamente se incrementa de ocho en los relatos iniciales a veinte en los finales. Las temáticas históricas incorporadas en esta sección continúan siendo las consecuencias que hasta el día de hoy existen en la sociedad chilena después del contexto histórico estudiado, tales como las violaciones a los derechos humanos, la desigualdad socio-económica, la relevancia de evitar el olvido, entre otras. Sin dudas, este aumento se debió a las conexiones explícitas entre pasado y presente que se trabajaron didácticamente en la sala de clases. Pero, a pesar de ello, se presentan dificultades didácticas en los aspectos de continuidad, ya que, en las palabras del grupo estudiantil, no tienen los conocimientos sobre los años 90 
y principios de los 2000 en Chile. Por ende, extrapolan de forma prácticamente intactas e inmutables las ideas impuestas por la dictadura cívico militar hasta el día de hoy.

Uno de los cambios más importantes evidenciados en las narraciones de los y las estudiantes se relaciona con el vínculo entre pasado y presente individual. En los relatos iniciales solo una estudiante se incorpora en el relato estableciendo una relación entre pasado e identidad personal. Esto último es una consecuencia de la validación de ciertos conocimientos en la escuela y el aula, en donde la subjetividad, la vida cotidiana y, por ende, los procesos de biografización de cada uno de los individuos quedan fuera (DeloryMomberger, 2014). Esto no solo niega la oportunidad de incorporar los contenidos y aplicar las habilidades del pensamiento histórico en los relatos autobiográficos, sino que también embarga la posibilidad que los educandos pueden pensar y reelaborar, a partir de la Educación histórica, su conciencia histórica y, por ende, también su actuar en sociedad. A ello se suma que la enseñanza de la Historia se ha centrado tradicionalmente en grandes personajes, los cuales a partir de sus acciones individuales influyen en los procesos históricos, situación que ha bloqueado la actuación de otros grupos, comunidades e intereses (González, Cerri \& Rosso, 2016), lo cual se extrapola a las personas comunes y corrientes como los estudiantes.

A pesar de la situación descrita, en las narraciones históricas finales se evidenciaron ciertos avances a la hora de vincular la identidad personal con los procesos históricos del pasado, ya que fueron identificados diez fragmentos con estas características. Esta situación se debió al trabajo sistemático y continuo, a partir de las habilidades históricas, principalmente la explicación histórica y la temporalidad, con los aspectos autobiográficos de los y las estudiantes. A ello, se sumó un proceso de reconocimiento y validación de sus experiencias, al incorporar sus relatos en el aula. Como plantea Wineburg (2001), "historical thinking, in its deepest form, is neither a natural process nor something that springs automatically from psychological development ${ }^{1}$ " (p. 7). De hecho, "goes against the grain of how ordinarily think, one of the reasons why it is much easier to learn names, dates, and stories than it is to change the basic mental structures we use to grasp the meaning of the past ${ }^{2 \prime}$ (p. 7). En esta línea, es relevante considerar que incorporar relatos autobiográficos en el aula y reelaborarlos a partir de las habilidades del pensamiento histórico tampoco es natural, sino que es necesario reconocer la conciencia histórica que trae el sujeto y, posteriormente, validarlas a través de su trabajo con las habilidades del pensamiento histórico.

Se ha planteado la importancia de que los y las estudiantes deberían tener una panorámica general del pasado (Kitson et al. 2015; Barca, 2013), a lo que hay que sumar la comprensión del pensamiento histórico desde una perspectiva dialéctica, lo cual se trata de "comprender el mundo en término de interconexiones, los vínculos entre las cosas tal como son ahora, sus propias precondiciones (es decir, de dónde vienen), y sus posibilidades de futuro" (Ross \& Gautreaux, 2018, p. 385). Esta forma de entender el pensamiento histórico

1 El pensamiento histórico, en su forma más profunda, no es un proceso natural ni algo que surja automáticamente del desarrollo psicológico (traducción propia).

2 Va contra la corriente de lo que normalmente se piensa, lo cual es una de las razones porque es mucho más fácil aprender nombres, fechas e historias que cambiar las estructuras mentales básicas que usamos para captar el significado del pasado (traducción propia). 


\section{А. F. Sото}

podría entregarles espacios y posibilidades a los educandos para que en la sala de clases, a partir de las habilidades asociadas con la Educación histórica, puedan elaborar procesos de biografización. Para ello, es necesario considerar el carácter liminal (Plá, 2012) de la didáctica de la Historia y las Ciencias Sociales, ya que convive con la sociología educativa, el análisis del discurso político educativo e incluso, considerando esta investigación, con las narrativas autobiográficas de los y las estudiantes.

Por último, un aspecto nuevo es que la voz del estudiante también se hace presente para proyectar el futuro social de Chile y las acciones que realizaría como agente político para construirlo. En los relatos iniciales no se encontró ningún fragmento con esas características, en comparación con los catorce presentes en los relatos finales, en donde los estudiantes están conscientes de esa posibilidad, "como la conciencia de la libertad para decidir cuál es el futuro que deseamos” (Santisteban \& Anguera, 2014, p. 254).

\section{CONCLUSIONES}

La presente investigación tuvo como objetivo principal analizar las transformaciones y continuidades de las voces presentes en los relatos de los y las estudiantes sobre el antes, el durante y el después de la dictadura cívico militar en Chile, luego de ser expuestas a una secuencia didáctica que buscó elaborar narrativas históricas con rasgos autobiográficos. Con ello, se pretendió rescatar el proceso de enseñanza-aprendizaje y no solo un momento particular de la intervención. A raíz de lo anterior, se puede concluir que las voces presentes en las narraciones son diversas, presentan tensiones entre ellas y pueden variar dependiendo del proceso didáctico implementado.

Una de las voces que persiste durante toda la intervención didáctica es la del narrador omnisciente, figura utilizada por los estudiantes con la idea de narrar el pasado bajo un manto de objetividad. Esta forma de relatar es muy común en las narraciones y evidenció que este grupo estudiantil, con ciertas temáticas, entiende el pasado como una verdad, la cual no deja espacios para voces alternativas, conflictivas o contradictorias. Situación que demuestra la escasa conciencia de las habilidades y finalidades del pensamiento histórico, a lo que se suma la asimetría de este pasado objetivo con los conocimientos obtenidos en los espacios informales de aprendizaje.

Además de lo anterior, uno de los cambios presentes en los relatos finales es la incorporación de las voces de personas significativas para los y las estudiantes como un complemento a lo relatado por el narrador omnisciente, otorgándoles más validez que a los documentos trabajados durante la intervención didáctica. Situación que no es extraña, ya que una característica que se mantiene durante toda la implementación es la resistencia para incorporar voces alternativas y conflictivas provenientes de fuentes primarias y secundarias.

Al igual que en los relatos iniciales, en las narraciones históricas con rasgos autobiográficos finales, continua emergiendo la voz de los educandos en la evaluación de temáticas históricas conflictivas, pero en menor cantidad que en un inicio. Ello en contraste con las transformaciones más relevantes, relacionadas con la aparición de la voz del grupo 
estudiantil en las relaciones entre pasado, presente y futuro, desde una perspectiva social e individual. Lo nuevo de esto último es que el grupo estudiantil pudo, en la etapa final de la intervención, conectarse subjetivamente con lo trabajado en el aula. En otras palabras, se creó una vinculación dialéctica entre el pasado y la construcción identitaria de los individuos, la cual permitió construir una oportunidad para que los educandos experimenten el pasado y las habilidades del pensamiento histórico como un insumo que podría ser utilizado para reelaborar su relato autobiográfico. Proceso que se logró después de trabajar de forma sistemática y constante los relatos confeccionados por los alumnos durante todo el proceso didáctico.

A pesar de lo anterior, una de las debilidades del estudio es el número de estudiantes que logró realizar todas las actividades en los tiempos destinados en la sala de clases, razón por la cual sus resultados no son generalizables. Además, se evidenciaron complejidades en la elaboración de narraciones históricas con rasgos autobiográficos porque el grupo estudiantil no estaba acostumbrado a incorporar su subjetividad y fueron reticentes a ponerlas en tensión en un espacio que tradicionalmente las excluye. A ello se suma que las actividades que constantemente realizan en la clase se encuentran estructuradas y esquematizas por el docente, lo cual provoca inseguridades a la hora de utilizar las habilidades históricas en la construcción de relatos más largos, además de usarlas para evaluar las narraciones elaboradas por sus personas significativas o por ellos mismos. Esto último es vital a la hora de aplicar este tipo de habilidades en instancias lejanas al aula, en donde los alumnos de forma autónoma y sin intermediación del profesor deberían realizar este ejercicio. Por ello, si se pretende que los y las estudiantes utilicen las habilidades del pensamiento histórico para actuar como ciudadanos democráticos, es imprescindible trabajar didáctica y dialécticamente la cultura histórica, la conciencia histórica y los relatos autobiográficos que han ido elaborando en su vida.

\section{REFERENCIAS}

Barca, I. (2013). Conciencia histórica: pasado y presente en la perspectiva de los jóvenes en Portugal. Clio y Asociados, 17, 16-26.

Barton, K. (2010). Investigación sobre las ideas de los estudiantes acerca de la Historia. Enseñanza de las ciencias sociales: revista de investigación, 9, 97-113.

Bolívar, A., \& Domingo, J. (2006). La investigación biográfica y narrativa en Iberoamérica: Campos de desarrollo y estado actual. Forum: Qualitative Social Research, 7(4), Art. 12. Recuperado de: https://www.qualitative-research.net/index.php/fqs/article/ view/161/358

Cataño, C. (2011). Jorn Rusen y la conciencia histórica. Historia y Sociedad, 21, 223-245.

Cerri, L., De Oliveira, J., \& Cuesta, V. (2014) Conciencia histórica y representaciones de identidad política de jóvenes en el Mercosur. Enseñanza de las ciencias sociales: revista de investigación, 13, 3-15.

Delory-Momberger, C. (2014). Experiencia y Formación. Biografización, biograficidad y heterobiografía. RMIE, 19(62), 695-710. 


\section{А. F. Sото}

Delory-Momberger, C. (2015). El relato de sí como hecho antropológico. En G. Murillo (Comp.), Narrativas de experiencia en educación y pedagogía de la memoria. (pp. 57-68) Buenos Aires: Editorial de la Facultad de Filosofía y Letras, Universidad de Buenos Aires. http:// doi.org/10.2307/j.ctvtxw30v.5

González, J. (2009). Historias de vida y teorías de la educación: tendiendo puentes. Cuestiones Pedagógicas, 19, 207-232.

González, P., \& Henríquez, R. (2003). Conciencia histórica y cultura material: aproximaciones desde la enseñanza de la historia. En E. Ballesteros, C. Fernández, J. Molina y P. Moreno (Coords.), El Patrimonio y la didáctica de las Ciencias Sociales (113-122), Cuenca: AUPDCS.

González, F., Cerri, L., \& Rosso, A. (2016). Héroes y cultura histórica entre estudiantes en Chile. Revista Brasileira de História, 36(71), 1-24.

Henríquez, R. (2009). Aprender la historia ajena. El aprendizaje y la comprensión histórica de alumnos inmigrantes en Catalunya. Enseñanza de las ciencias sociales: revista de investigación, 8, 45-54.

Henríquez, R. (2013). Las habilidades para representar: explicar y narrar el pasado. En Muñoz y Osandón (Comp.), La didáctica de la Historia y la formación de ciudadanos competentes (pp.181-202). Santiago de Chile: DIBAM.

Henríquez, R., Carmona, A. Quinteros, A., \& Garrido, M. (2018). Leer y escribir para aprender Historia. Secuencias para la enseñanza y el aprendizaje del pensamiento histórico. Santiago de Chile: Ediciones UC.

Hernández, R., Fernández, C., \& Baptista, P. (2010). Metodología de la investigación (5ª ed.). México: Mc Graw Hill.

Kitson, A., Steward, S, \& \& Husbans, C. (2015). Didáctica de la Historia en Secundaria Obligatoria y Bachillerato (Pablo Manzano Bernárdez, trad.). España: Ediciones Morata.

Krippendorf, K. (1990). Metodología de análisis de contenido. Teoría y Práctica. Barcelona, España: Paidós Comunicación.

Merchán, F. (2010). La práctica de la enseñanza de las Ciencias Sociales como objeto de investigación de la Didáctica: importancia y dificultades metodológicas. Bases para una teoría de la acción en el aula. En R. Ávila, M. Rivero y P. Domínguez (Coords.), Metodología de investigación en Didáctica de las Ciencias Sociales (pp. 105-113). Zaragoza.

Ministerio de Educación. (2015). Historia, Geografía y Ciencias Sociales. Programa de Estudio Tercer año medio. Actualización 2009.

Passegi, M. (2011). Aproximaciones teóricas a las perspectivas de investigación (auto) biográfica en educación. Revista Educación y Pedagogía, 23(61), 25-40.

Plá, S. (2005). Aprender a pensar históricamente. La escritura de la historia en el bachillerato. México: Plaza y Valdés.

Plá, S. (2012). La ilusión científica de la didáctica de la historia. Provocaciones teóricas sobre el conocimiento histórico escolar. En J. Blázquez, P. Latapí y H. Torres. (Coords.), Memoria del Cuarto Encuentro Nacional de Docencia, Difusión y Enseñanza de la Historia. Segundo Encuentro Internacional de Enseñanza de la Historia. Tercer Coloquio entre tradición y modernidad, México: Reddieh. 
Plá S., \& Pérez, M. (2013). Pensar históricamente sobre el pasado reciente en México. Clío \& Asociados, 17, 27-55.

Plá S., \& Pagès, J. (2014). Una mirada regional a la investigación en enseñanza de la historia en América Latina. En S. Plá y J. Pagès. (Coords.), La investigación en la enseñanza de la historia en América latina (pp. 13-38). México: Universidad Pedagógica Nacional.

Prego, S., \& Armas, X. (2014). “.... Y sometieron a los pueblos indios que había allí”. Competencia narrativa de estudiantes de bachillerato sobre la Historia de los Estados Unidos. En J. Pagés y A. Santisteban (Coords.), Una mirada al pasado y un proyecto de futuro: investigación e innovación en didáctica de las Ciencias Sociales (pp. 727-734). Barcelona: Simposio Internacional de Didáctica de las Ciencias Sociales.

Ross, W., \& Gautreaux, M (2018). Pensando de manera crítica sobre el pensamiento crítico. Aula Abierta. 47(4), 383-386.

Rusen, J. (1992). El desarrollo de la competencia narrativa en el aprendizaje histórico. Una hipótesis ontogenética relativa a la conciencia moral'. Propuesta Educativa, 7, 27-36.

Rusen, J. (2013). Tiempo en ruptura. México: Universidad Autónoma Metropolitana.

Salazar, J. (2006). Narrar y aprender historia. México: Universidad Nacional Autónoma de México.

Sánchez, F. (2009). Cultura Histórica. [online] Culturahistorica.org. Recuperado de http:// www.culturahistorica.es/sanchez_marcos/cultura_historica.pdf

Santisteban, A. (2010). La formación de competencias de pensamiento histórico. Clío \& Asociados, 10, 34-56.

Santisteban, A., \& Pagès, J. (2014). ¿Qué sabemos sobre la enseñanza y el aprendizaje de la Historia en España. 20 años de investigación? En S. Plá y J. Pagès (coord.), La investigación en la enseñanza de la historia en América latina (pp. 155-192). México: Universidad Pedagógica Nacional.

Sanstisteban, A., \& Anguera, C. (2014). Formación de la conciencia histórica y educación para el futuro. Clío \& Asociados, 18-19, 249-267.

Schmidt, M. (2014). Cultura histórica e aprendizagem histórica. Revista NUPEM, 6(10), 31-50.

Schmidt, M. (2016). Jovens brasileiros, consciencia histórica e vida prática. Revista História Hoje, 5(9), 31-48.

Wineburg, S. (2001). Historical thinking and other unnatural acts: charting the future of teaching the past. Philadelphia: Temple University Press. 